\title{
Avant-propos : Les enfants sacrifiés
}

\section{Thomas Keller}

\section{(2) OpenEdition}

\section{Journals}

Édition électronique

URL : http://journals.openedition.org/ceg/1909

DOI : 10.4000/ceg.1909

ISSN : 2605-8359

Éditeur

Presses Universitaires de Provence

Édition imprimée

Date de publication : 15 juin 2014

Pagination : 7-25

ISSN : 0751-4239

Référence électronique

Thomas Keller, «Avant-propos : Les enfants sacrifiés », Cahiers d'Études Germaniques [En ligne], 66 |

2014, mis en ligne le 17 décembre 2017, consulté le 22 novembre 2020. URL : http://

journals.openedition.org/ceg/1909; DOI : https://doi.org/10.4000/ceg.1909 


\title{
Avant-propos Les enfants sacrifiés
}

\begin{abstract}
Pendant qu'on enseignait le meurtre du père, quelques personnes, de par le monde, décidaient, pendant la Première et la Seconde Guerre mondiale, de mettre à mort des dizaines de millions de jeunes gens, qui n'avaient pas plus de vingt-cinq ans. Au moment même où on enseignait le meurtre du père, ces guerres témoignaient du meurtre du fils... Qu'est-ce qu'une déclaration de guerre ? C'est un vieillard, quelque part, une sorte d'ignoble grand-père qui dit à son collègue, vieux père, de l'autre côté de la frontière : "Sois gentil. Avec tes enfants, tue les miens. À charge de revanche ». Voilà l'idée que je me fais de la guerre : un atroce sacrifice d'innombrables enfants..

Si j'ai raison de définir la guerre comme le meurtre des fils, alors il n'y a jamais eu de guerre de la France contre l'Allemagne...'
\end{abstract}

Peut-on parler ainsi de la Première et de la Deuxième Guerre mondiale ? Les propos de Michel Serres peuvent paraître trop globalisateurs et quelque peu naïfs. Ils semblent réunir deux guerres très différentes et occulter l'abîme séparant les envahisseurs de ceux qui doivent se défendre. Ils n'en posent pas moins deux questions cruciales, celle du caractère sacrificiel des cultures et celle de leur imbrication. Quelle est la culture qui serait prête à sacrifier ses enfants dans une guerre? Quelle est la culture pour laquelle des millions de personnes seraient prêtes à se sacrifier? Il est un peu trop simple de faire de la guerre, à l'instar de nombreux intellectuels et artistes, l'affaire des autres. Si l'on retourne le mot fameux de Carl Sandburg « Sometime they'll give a war and nobody will come» (souvent attribué à tort à Brecht « Stell dir vor, es ist Krieg und keiner geht hin »), on obtient: «Imagine-toi qu'ils fassent la guerre et que tous les jeunes y aillent ». Ainsi définit-on beaucoup mieux le lien étroit qui relia culture et violence lors de la guerre de 1914-18. M. Serres souligne l'action croisée de la France et de l'Allemagne. Une culture a besoin de l'autre pour mener à bien son œuvre meurtrière, pour s'affirmer. Elle fait tuer ses enfants par l'autre et elle tue les enfants de l'autre. Les enfants sont le médium d'une entente sordide.

Qui est ce vieillard "ignoble » de la culture ou de la civilisation (nous faisons ici abstraction de la distinction entre les deux) ? Quelle est la structure qui paraît être moins parricide, c'est-à-dire œdipienne, qu'infanticide ? Les archives culturelles mettent à notre disposition plusieurs récits narrant le

\footnotetext{
${ }^{1}$ Michel SERRES, Petites chroniques du dimanche soir 2, février 2006-mars 2007, Paris, Le Pommier, 2007, p. 30-31.
} 
meurtre de l'enfant, par exemple Saturne dévorant ses enfants, le meurtre des nouveau-nés perpétré par Hérode ou encore Abraham prêt à sacrifier son fils Isaac. Le mythe de Médée tuant ses enfants établit le lien entre la confrontation de deux cultures et le sacrifice de l'enfant.

Max Ernst transpose le meurtre de l'enfant au contexte franco-allemand. Dans l'un de ses montages, l'artiste franco-allemand relie la Première Guerre mondiale à la scène biblique $\mathrm{du}$ 《massacre des innocents $»^{2}$. Le titre fait explicitement allusion au récit narrant le meurtre des enfants ordonné par Hérode pour tuer le futur roi des juifs. M. Ernst insère dans son collage des personnes en fuite. Elles rappellent celles que l'on trouve dans les toiles de Nicolas Poussin représentant Le Massacre des innocents. Là où les tableaux de N. Poussin mettent en scène trois acteurs - la mère, l'enfant, les bourreaux ,$- \mathrm{M}$. Ernst réduit les mères qui s'enfuient à des schèmes et évacue toute action violente entre êtres humains. L'absence de victimes dans le tableau de M. Ernst est frappante. Aucun nouveau-né n'y est représenté. L'impression qui se dégage de l'œuvre est pourtant terrifiante. Le déchaînement de violence est imminent. L'être volant, qui rappelle un appareil construit par Lilienthal, renvoie également au mythe d'Icare. Icare est trahi par Dédale; les inventions de son père le tuent. L'être hybride, technique et humain, est décentré tandis que le centre de l'œuvre reste vide, aucun être humain n'y figure. Il s'offre au spectateur, il l'engloutit. Le spectateur doit s'imaginer les cibles mentionnées dans le titre : les innocents. La photo montre au centre la zone industrielle de la ville de Soissons détruite par l'aviation allemande; elle est empruntée à la publication Deutsches Kriegsflugwesen (1917). Les victimes sont invisibles. Le lien qui existe avec les jeunes gens dans les tranchées est toutefois facile à établir : les nouveau-nés, absents de l'œuvre, sont destinés à fournir le matériel humain devant être sacrifié dans cette guerre. Pour autant, le spectateur n'occupe ni la place du meurtrier, ni celle de la victime, ni celle des deux à la fois. Le tableau refuse la structure binaire. Le combat qui est représenté n'est engagé ni entre deux armées, ni entre deux pays. L'entrecroisement des configurations culturelles est invisible, il n'en demeure pas moins fondateur. L'image fait entrevoir la guerre en tant que configuration mentale. Le propre de la culture infanticide consiste à transformer le lien entre deux cultures en une machine guerrière, celle du meurtre des innocents. Le tableau de M. Ernst remplace la guerre entre «Allemands » et « Français » par la représentation de la violence qui frappe sans aucune distinction les jeunes gens.

\footnotetext{
${ }^{2}$ L'œuvre est consultable sur Internet à l'adresse : http://www.zeitenblicke.de/2004/01/ derenthal/derentha19.htm.
} 


\section{Une violence peut en cacher une autre : le tiers déclenchant le conflit}

Les décennies d'avant 1914 connaissent déjà des tentatives visant à soumettre la coexistence des États européens à des règles. Cette œuvre civilisatrice s'inspire de l'esprit des Lumières, entre autres des conceptions kantiennes de la paix. Imposer aux États-nations une juridiction universelle doit permettre de réduire la tendance à la violence. Cet idéal de paix requiert un certain renoncement à la souveraineté en faveur de l'établissement d'instances internationales, mais il repose toujours sur la coopération entre États souverains. Dès 1864, les conventions de Genève soumettent le traitement des blessés, des prisonniers de guerre et des civils à des normes ; les conventions de La Haye de 1899 et de 1907 ont pour objectif le désarmement et la prévention de la guerre. Ce processus, qui inaugure une politique intérieure mondiale, se poursuit après la Première Guerre mondiale avec la fondation de la Société des Nations et le Pacte Briand-Kellog de 1928 qui veulent bannir la guerre. Toutes ces codifications des rapports entre nations s'élaborent avec la participation de la France et de l'Allemagne. Elles s'appliquent à des conflits armés internationaux qui ont pour acteurs des États. La Première comme la Deuxième Guerre mondiale illustrent ainsi la violation par un État souverain de principes universels instaurés par le droit international. Le traité de paix, qui établit les responsabilités, détermine la différence entre les défenseurs de la paix et de la souveraineté d'une part et l'agresseur de l'autre. La guerre n'est juste que quand elle défend des valeurs universelles.

Si les tentatives de soumettre à des règles les conflits interétatiques représentent un progrès certain, elles occultent pourtant une autre réalité. Les traités eux-mêmes sont souvent source de conflits. De la paix de Westphalie (1648) au premier traité de Paris en 1814, tous les traités de paix connaissent l'oubli et l'amnistie. Le terme de « sécurité » n'y apparaît pas. Le premier traité qui fut signé entre une monarchie et une république, à savoir le traité de Bâle (1795) entre la Prusse et la République française, obéit lui aussi à cette règle. Ce traité met pour la première fois en place, conformément aux conceptions kantiennes, un mécanisme tiers destiné à assurer la paix. Toutefois, l'organisation de la paix, bien qu'elle soit par définition improvisée ${ }^{3}$, divise les belligérants de plus en plus en vainqueurs et vaincus.

La rupture intervient après les Cent-Jours et la défaite de Waterloo : le deuxième traité de Paris, en 1815, introduit alors des nouveautés. Certes, la

\footnotetext{
3 « Entre les deux extrêmes, clémence absolue et sévérité inflexible, les vainqueurs n'ont cessé de balancer », "Introduction », in Jean-Paul CAHN, Françoise KNOPPER, Anne-Marie SAINT-GILLE (dir.), De la guerre juste à la paix juste. Aspects confessionnels de la construction de la paix dans l'espace franco-allemand ( $X V I^{e}-X X^{e}$ siècle), Villeneuve d'Ascq, Presses Universitaires du Septentrion, 2008, p. 7-18, ici p. 9. Voir aussi Françoise KNOPPER, «Les traités de paix, des tournants? », Revue d'Allemagne et des pays de langue allemande, 2011, p. $163-318$.
} 
France a été invitée à la table des négociations, ses pertes territoriales restent limitées. Mais Napoléon est désigné comme le coupable, la France doit payer des réparations, des territoires français restent occupés pendant quelques années. Les peines ne frappent pas le régime napoléonien mais le régime suivant. La Sainte-Alliance formée de monarchies crée un équilibre européen assurant la paix pendant un demi-siècle. Mais dorénavant les relations interétatiques sont contaminées par une mimésis de la violence.

Les traités de paix suivants réitèrent le scénario. Après la défaite de 1871, la France, qui n'est plus une monarchie, doit payer des réparations. L'occupation de territoires français ne s'arrête pas avec la fin de la guerre. Lors de la signature du traité de Versailles, les alliés vainqueurs déclarent agir au nom de l'humanité, au nom des pays civilisés. L'Allemagne est exclue des négociations. L'Empire allemand a détruit l'équilibre européen et la paix. Il n'est pas seulement désigné comme coupable, mais comme le seul coupable. L'Allemagne est condamnée à payer des réparations, la rive gauche du Rhin est occupée. Les conséquences frappent la République de Weimar. Les mécanismes de sécurité, comme le désarmement de l'Allemagne par exemple, deviennent l'affaire de la Société des Nations, censée contrôler la situation.

On assiste à l'introduction d'un tiers qui, en induisant une asymétrie, rend le vaincu méchant (un constat qui bien évidemment n'excuse nullement les inhumanités à venir). L'ordre d'après-guerre soumet également jeunes Français et jeunes Allemands, qui ont combattu dans les tranchées, à une dichotomie et une hiérarchisation problématiques. Il les divise en vainqueurs et vaincus, alors que leur façon d'être et de penser se dérobe le plus souvent à toute dualité entre pays. Tandis que les traités, les cérémonies et les discours officiels entretiennent les mécanismes belligènes, les morts et beaucoup de survivants démentent les binarités. S'il est vrai que le poilu ou le Kriegsteilnehmer devenu victime n'est pas automatiquement «bon » et pacifiste, il devient toutefois, en quelque sorte, étranger à la communauté nationale.

La guerre aussi est un type d'interaction réglementé. Bien qu'elle puisse paraître en être le type le plus extrême ou le plus destructeur, la guerre obéit, plus que tout autre conflit, à une codification. Son caractère meurtrier provoque des réactions tendant à soumettre les situations de crise à des règles. Pendant l'ère des nations, les États souverains ont imposé des garanties grâce auxquelles les combattants bénéficient d'une certaine protection (par exemple le statut de prisonnier de guerre). Lors de conflits violents qui ne connaissent pas l'état de guerre déclarée entre deux entités, une partie tend à réclamer pour elle le droit, l'humanité et la volonté de paix, et à qualifier l'autre partie de meurtriers, de terroristes, de pirates etc. Cet autre dévalorisé ne jouit plus de tous les droits. La Première Guerre mondiale ne semble pas engendrer une telle inégalité entre combattants. Elle est un conflit symétrique entre deux États. Or cette guerre expose les jeunes victimes à une violence extrême. Ils sont, dans leur chair même, des tiers qui relient les États belligènes et échappent en même temps à la souveraineté nationale. 
Une historiographie croisée permet de percevoir plus aisément le lien entre différend et réconciliation. En cela, elle rappelle le mot d'Héraclite selon lequel « Il faut savoir que la guerre est commune, et la justice discorde, et que tout advient selon dispute et nécessité ». Elle favorise aussi la logique de l'amnistie. Le commentaire de Plutarque sur la loi de Solon interdisant de dire du mal des morts, " il est possible d'ôter à la haine son éternité (La vie de Solon) ${ }^{4}$ dont le but est de mettre fin à la guerre civile à Athènes, constitue le premier décret d'amnistie. Il vise à empêcher que la haine ne s'installe. Mais cette logique ne nie pas le conflit. Elle évite de porter un jugement moral sur les parties qui s'opposent. Les penseurs contemporains qui réhabilitent l'art éristique des sophistes, Barbara Cassin en France ${ }^{5}$, Rüdiger Bubner ${ }^{6}$ en Allemagne, n'appellent pas à rompre avec les mécanismes fondateurs de la paix dans la tradition kantienne. Mais ils tiennent compte de l'effet belligène des discours faisant de la violence l'affaire de l'autre.

Tout comme le discours sur l'ennemi héréditaire reproduit purement et simplement un rapport de forces, la guerre ne reflète pas forcément la pensée de ceux qui doivent la mener. Ils sont acteurs et victimes de la cruauté, celle de la Première Guerre mondiale comme celle des guerres antérieures, telle la guerre de Trente Ans, la guerre de Sept Ans, ou encore la campagne de Russie de Napoléon. Au premier abord, la Première Guerre mondiale semble représenter la confrontation entre États souverains et entre deux camps, avec une déclaration de guerre, des règles sur la conduite à suivre avec les prisonniers de guerre. La guerre se termine par un traité de paix. Pour autant, les analyses récentes dévoilent surtout le sacrifice des innocents, qui les soustraie à la souveraineté 7 . Elles révèlent l'existence d'expériences et de discours transculturels dépassant la frontière qui sépare les nations. C'est justement ce double caractère agonal, la violence de la machine de guerre et celle des réactions contre la culture infanticide, qui signale le dépassement de la binarité et les limites d'une écriture souverainiste des nations.

Certes, le conflit et tout particulièrement la guerre, présupposent un contact entre deux entités, entre deux cultures, entre deux pays, entre deux armées, entre deux personnes. Cependant, la Première Guerre mondiale dissout les appartenances claires et univoques. Il existe depuis longtemps pour Français et Allemands des zones de contacts, des migrations et des guerres déclenchant des imbrications. Les guerres franco-allemandes - Iéna/ Waterloo, Sedan, la «Grande Guerre », la Deuxième Guerre mondiale semblent suggérer une relation spécifique, un rapport d'«ennemis

\footnotetext{
${ }^{4}$ Barbara Cassin, Olivier CAYLa, Philippe-Joseph SAlazar (dir.), Vérité, réconciliation, réparation, Paris, Seuil (coll. «Le Genre humain »), 2004, p. 38.

${ }^{5}$ Ibid. ; Barbara CASSIN, « Amnestie und Vergebung. Für eine Trennung von Ethik und Politik », in Frank R. PFETSCH (dir.), Konflikt, Berlin, Springer, 2004, p. 129-148.

${ }^{6}$ Rüdiger BUBNER, "Von der Streitkunst der Sophistik zu modernen Konflikten », in PFETSCH, Konflikt, p. 101-108.

Niels Werber, "Erzählen im Krieg und Nicht-Krieg ", LiLi, Zeitschrift für Literaturwissenschaft und Linguistik, $42^{\mathrm{e}}$ année, $\mathrm{n}^{\circ}$ 165, Postsouveränes Erzählen, mars 2012, p. 87-105.
} 
héréditaires ». L'impression qui se dégage d'un siècle et demi d'hostilités fait pourtant oublier que les mécanismes transculturels sont particulièrement forts en période de conflit, de guerre ou d'occupation. La reconstitution patriotique des conflits fait aussi l'impasse sur la violence perpétrée par les belligérants à l'encontre de leur propre population, sur les désertions et les mutineries, surtout celles qui se produisent pendant la seconde moitié de la Première Guerre mondiale. L'apparition de morts victimes non de l'adversaire mais de leur propre camp, tels les fusillés pour l'exemple, caractérise en effet la seconde phase de la guerre ${ }^{8}$. La recherche récente jette un autre regard sur les morts, et aussi sur les survivants comme les gueules cassées et les traumatisés, souvent soupçonnés de simulation ${ }^{9}$.

L'historiographie croisée met en question les rétrospectives construisant, de 1914 à 1918, un patriotisme de tous les Français ou de tous les Allemands. Elle souligne le fait que les jeunes étaient victimes d'une violence systématique des deux côtés du front ${ }^{10}$. Pour la plupart des soldats, survivre était plus important que vaincre. À l'enthousiasme avaient succédé la fatigue, la désillusion et la révolte. Si la grande majorité des combattants voulaient, surtout à compter de 1917, tout simplement en finir avec la guerre, ils désiraient aussi rompre avec ce monde prêt à immoler toute une génération. Il s'agit là d'une position croisée qui transforme, dans les deux pays, les jeunes envoyés au combat en victimes des forces coercitives internes. Contrairement à la Deuxième Guerre mondiale, guerre incontournable pour venir à bout du nazisme, les objectifs de la guerre de 14-18 ne peuvent justifier les sacrifices subis. Tout comme la guerre ne résout rien, le pacifisme n'abolit en rien les conditions qui conduisent à accepter la mort de millions de jeunes gens. La question de la violence reste ainsi d'actualité. Les deux guerres mondiales et la guerre civile européenne sapent définitivement les fondements d'une écriture culturaliste et binaire des nations.

Toutefois, c'est aussi à travers l'agonalité que la guerre entre États expose ses contradictions. Le lien entre culture et violence se complique. S'il semble être le propre des élites politiques et militaires, il se manifeste aussi chez les personnes dont l'appartenance à un État et/ou à une nation n'est pas évidente,

\footnotetext{
${ }^{8}$ Ulrich BRÖCKLING, Michael SIKORA (dir.), Armeen und Deserteure. Vernachlässigte Kapitel einer Militärgeschichte der Neuzeit, Göttingen, Vandenhoeck \& Ruprecht, 1998 ; Christoph JAHR, Gewöhnliche Soldaten. Desertion und Deserteure im deutschen und britischen Heer 1914-1918, Göttingen, Vandenhoeck \& Ruprecht, 1998 ; Nicolas OFFENSTADT, 14-18 aujourd'hui. La Grande Guerre dans la France contemporaine, Paris, Odile Jacob, 2010 ; id., Les fusillés de la grande guerre et la mémoire collective (1914-1999), Paris, Odile Jacob, 1999 ; Jean-Yves LE NAOUR, Fusillés. Enquête sur les crimes de la justice militaire, Paris, Larousse, 2010.

9 Jean-Yves Le NAOUR, Le soldat inconnu vivant 1918-1942, Paris, Hachette, 2002; Id., Les soldats de la honte, Paris, Perrin, 2011; id., Les poilus, Paris, classiques Garnier, 2012.

${ }^{10}$ Nicolas BEAUPRÉ, Écrire en guerre, écrire la guerre (France, Allemagne 1914-1920), Paris, CNRS Éditions, 2006 ; id., Deutsch-französische Geschichte (Histoire franco-allemande) vol. VIII, Das Trauma des großen Krieges (1918-1932/33), Darmstadt, Wissenschaftliche Buchgesellschaft, 2009.
} 
voire contestée. Les dispositifs antagonistes mentaux et discursifs de l'avantguerre se retrouvent chez les intellectuels et artistes des deux pays, y compris parmi les protagonistes des médiations franco-allemandes. Les avant-gardes adressent une autre violence aux forces belligérantes. Cette autre violence qui est celle de la culture même se manifeste dans un contexte franco-allemand et révèle le potentiel conflictuel d'une conception et perception identitaires des individus et des nations. La mémoire officielle s'avère source de nouveaux conflits. Des situations fortement ambiguës caractérisent les territoires occupés.

\section{Cultures majoritaires - cultures minoritaires - cultures contestataires}

On interprète souvent les conflits entre la France et l'Allemagne à la lumière de l'opposition classique entre l'État-nation et la nation culturelle. Cependant, le conflit franco-allemand brouille ces lignes de démarcation. Certes, la Kulturkritik à l'allemande est souvent ethniciste. Il serait néanmoins réducteur de nier toute agonalité dans les prises de positions universalistes et d'assimiler toute conception culturaliste au bellicisme. L'idéologie nationale belliciste a une fonction intégratrice à l'intérieur; les groupes minoritaires y voient l'occasion d'adhérer pleinement à la nation. Le désir d'appartenir à la communauté, celui d'une intégration sans réserve, renforce le penchant vers un conflit mené contre l'ennemi extérieur.

Les discours valorisant la violence se retrouvent dans des familles politiques très diverses. La réaction enthousiaste à la guerre est commune aux représentants du système et aux contestataires bien que les raisons diffèrent. Il peut paraitre curieux que le néokantisme participe à la mobilisation de part et d'autre de la frontière. On la rencontre chez les néokantiens Hermann Cohen et Léon Brunschvicg. Leurs " adversaires » dans le champ disciplinaire, les vitalistes Eucken, Scheler et Bergson, se font également les chantres de la guerre. Les sociologues n'échappent pas à ce nivellement des différences disciplinaires et politiques comme le montrent par exemple les écrits de guerre de Durkheim ou de Simmel.

L'espoir que la guerre puisse renouveler une civilisation sclérosée se retrouve chez des penseurs français et allemand, indépendamment de leur appartenance aux courants nationalistes ${ }^{11}$. Ce sont précisément ces penseurs

\footnotetext{
${ }^{11}$ Christophe Prochasson, Anne RASMussen, Au nom de la patrie, les intellectuels et la première guerre mondiale 1910-1919, Paris, La Découverte, 1996 ; Wolfgang J. MOMMSEN (dir.), Kultur und Krieg. Die Rolle der Intellektuellen, Künstler und Schriftsteller im Ersten Weltkrieg, München, Oldenbourg, 1996 ; Jürgen VON UNGERN-STERNBERG, Wolfgang VON Ungern-SternBerg, Der Aufruf « An die Kulturwelt! ». Das Manifest der 93 und die Anfänge der Kriegspropaganda im Ersten Weltkrieg. Mit einer Dokumentation, Stuttgart, Reclam, 1996 ; Jeffrey VERHEY, Der "Geist von 1914" und die Erfindung der Volksgemeinschaft, Hamburg, Hamburger Edition, 2000 ; Barbara BESSLICH, Wege in den Kulturkrieg. Zivilisationskritik in Deutschland 1890-1914, Darmstadt, Wissenschaftliche Buchgesellschaft, 2000 ; Kurt FLASCH,
} 
qui émettent des critiques sur leur propre pays, sur la nation embourgeoisée si méprisée par Péguy ou par Scheler qui, en 1914, en viennent à célébrer la guerre.

L'attitude des penseurs anti-utilitaristes des deux pays est particulièrement inspirée par l'espoir d'une rupture, d'un dépassement du matérialisme par la guerre. Nationalismes de la culture majoritaire et aspirations des cultures contestataires se conjuguent donc pour abaisser le seuil menant à la violence. Ce goût pour l'agonal dirigé contre la Troisième République caractérise « l'avant-garde littéraire de la nouvelle France » (« die literarischen Wegbereiter des neuen Frankreich », Curtius ${ }^{12}$ ) - Claudel, Gide, Péguy, Rolland, Suarès. Péguy est dreyfusard, mais en 1914 il peste contre le pacifisme de Jaurès. Les non-conformistes anti-idéalistes et anti-utilitaristes des deux pays se ressemblent de plus en plus ${ }^{13}$.

Les discours agonistiques se multiplient aussi dans les arts, les lettres et les sciences humaines dès avant 1914. Leurs auteurs sont souvent critiques à l'égard des systèmes en place. Dans les disciplines telles que la géographie, la sociologie ou encore l'ethnologie, les concepts identifient l'agonalité dans les contextes extra-européens et européens. L'enthousiasme pour la guerre des anarcho-syndicalistes et des dreyfusards, d'un Grave et d'un Péguy, l'idolâtrie de la guerre d'un Simmel ou d'un Scheler, le Oui au combat des artistes comme celui prôné par Georg Heym ou Guillaume Apollinaire démentent l'existence d'une opposition entre un monde politique qui serait militarisé et un monde culturel qui serait pacifique.

Il s'ensuit que les concepts permettant le dialogue entre Allemands et Français sont justement des concepts agonaux. La situation est paradoxale et médiatrice: les uns voudraient combattre le voisin pour appartenir à la nation, les autres combattre leur propre nation dans le combat contre l'autre.

\section{De la guerre fantasmée à la guerre réelle}

Le bellicisme n'est pas uniquement le fait des élites militaires et politiques. Le sujet abordé dans ce recueil requiert le concours de spécialistes de l'histoire, de la littérature, de la culture et des sciences humaines. L'ensemble des contributions s'élève contre l'idée réductrice selon laquelle

Die geistige Mobilmachung. Die deutschen Intellektuellen und der Erste Weltkrieg, Ein Versuch, Berlin, Alexander Fest, 2000 ; Matthias SCHÖNING, Versprengte Gemeinschaft. Kriegsroman und intellektuelle Mobilmachung in Deutschland 1914-1933, Göttingen, Vandenhoeck \& Ruprecht, 2009 ; Wolfram PYTA, Carsten KRETSCHMANN (dir.), Burgfrieden und Union sacrée. Literarische Deutungen und politische Ordnungsvorstellungen in Deutschland und Frankreich 1914-1933, München, Oldenbourg, 2011.

${ }_{12}$ Ernst Robert CURTIUS, Die literarischen Wegbereiter des neuen Frankreich, Potsdam, G.Kiepenheuer, 1923.

${ }_{13}$ Pour ce qui concerne la question de la «troisième intelligence », cf. Thomas KeLLER, Deutsch-Französische Dritte-Weg-Diskurse. Personalistische Debatten der Zwischenkriegszeit, München, Fink, 2001, p. 47-98. 
les historiens proposeraient une reconstitution authentique des hostilités militaires et les spécialistes de la littérature ne s'appuieraient que sur des documents fictionnels et se feraient davantage l'avocat des victimes. Le présent volume lit l'expérience de la guerre comme narration historique et artistique.

Point n'est besoin d'attendre août 1914, c'est-à-dire la ferveur guerrière généralisée du début de la guerre, pour que se révèlent les orientations bellicistes présentes dans tous les pays européens. Des études récentes montrent des convergences remontant au XIX ${ }^{\mathrm{e}}$ siècle $^{14}$. L'anticipation et l'imagination de la guerre font partie du climat d'une époque marquée par le darwinisme social, qui légitime la survie et l'expansion de la nation forte. Les pangermaniques s'enthousiasment pour le Volkskrieg; les nationalistes de l'Action Française et de la génération Agathon, qui combattent la Troisième République "sous influence allemande», prêchent une idéologie belliciste. Le milieu artistique français et le milieu universitaire allemand (par exemple Troeltsch et Delbrück) sont particulièrement sensibles aux concepts agonaux. A ce titre, un cap est franchi en 1911 (cf. la contribution de Gangolf Hübinger).

Les représentants des différentes disciplines n'échappent pas à la mobilisation, ni les théologiens protestants et catholiques en France et en Allemagne, ni les géographes qui anticipent et mènent la guerre en visualisant des objectifs au moyen de la cartographie. Les Considérations d'un apolitique de Thomas Mann, qui établissent la polarité entre le monde germanique (protestant) et le monde latin (catholique), les propos d'un Durkheim, représentant l'esprit laïciste de la Troisième République, et la défense de la France par le catholique Claudel ne surprennent pas, compte tenu de l'appartenance de leurs auteurs à un milieu constitutif de la nation. Cependant, parmi les tenants d'une guerre à même de porter le coup fatal soit à la République embourgeoisée, soit à l'Empire wilhelminien matérialiste voire aux deux -, nombreux sont ceux qui s'intéressent aux affinités électives avec le voisin. Les artistes qui ont séjourné chez ce dernier peu de temps avant l'éclatement des hostilités (les Allemands à Paris, les Français à Munich ou à Berlin) et s'y sont fait des amis, ont besoin eux aussi d'un adversaire dans cette guerre qui doit permettre de se libérer du monde d'hier.

Les prises de position des protagonistes médiateurs des relations francoallemandes, Boutroux, Gide, Julius Meier-Graefe, Otto Grautoff ou encore Oskar Schmitz (Barbara Besslich), en témoignent. Les représentants de la Nouvelle Revue Française ne font pas exception à la règle qui consiste à rejoindre 1'Union sacrée ${ }^{15}$. Peintres et écrivains expressionnistes se portent volontaires au début de la guerre.

\footnotetext{
14 Un exemple : Jörn LeONHARD, Bellizismus und Nation, Kriegsdeutung und Nationsbestimmung, Europa und die Vereinigten Staaten 1750-1914, München, Oldenbourg, 2008.

15 Yaël DAGAN, La NRF entre guerre et paix 1914-1925, Paris, Tallandier, 2008.
} 
Le bellicisme des penseurs issus de minorités (catholiques et juifs en Allemagne, protestants et juifs en France) s'explique aussi par le désir d'appartenir pleinement à la nation. On peut en outre observer contrairement ou parallèlement à l'intégration infra-culturelle l'adhésion de «minoritaires 》 et " d'outsiders » acclamant la violence: Blaise Cendrars, originaire de Suisse, et Guillaume Apollinaire, de mère polonaise et de père inconnu, se rallient à la cause française (Joseph Jurt), le luxembourgeois Norbert Jacques à la cause allemande. Ils s'intègrent par le biais d'un discours antagoniste qui prend parti pour l'un des deux belligérants (Françoise Knopper). Ils sont des tiers potentiels qui refusent d'endosser le rôle du médiateur. Les reportages publiés dans les différents journaux - la Frankfurter Zeitung, la Neue Freie Presse, le Berliner Tageblatt et les Weisse Blätter - reflètent pourtant la diversité des stratégies des journalistes. Alice Schalek reprend les discours qui exaltent la guerre. Le nationaliste Norbert Jacques cherche les sensations fortes. Les articles parus dans le Berliner Tageblatt font preuve de plus de distance, ceux des Weisse Blätter sont pacifistes.

Le regard porté sur les tranchées fait souvent oublier que, pendant la Première Guerre mondiale, une partie de la population française vit quatre années durant sous occupation allemande. Depuis quelque temps l'intérêt des chercheurs se porte sur la vie derrière le front entre 1914 et $1918^{16}$. Les frontières sont floues entre résistance et collaboration, entre patriotisme et trahison. La réalité se situe souvent entre les deux pôles. Les quatre années de « cohabitation » entre soldats allemands et civils français créent une intimité qui imprime sa marque. Les deux souverainetés, allemande et française, sont brouillées : les coopérations, les arrangements, le fonctionnement des services comme la poste, le téléphone et la presse altèrent la souveraineté nationale. Un journal comme La Gazette des Ardennes interprète certes favorablement le système politique, social et économique allemand, mais n'en est pas pour autant uniquement collaborationniste (David Weber). Ces ambiguités se réitèrent lors de l'occupation de la rive gauche de l'Allemagne par les troupes françaises après la Première Guerre mondiale. Les diatribes allemandes contre « la honte noire » révèlent toutefois un climat particulièrement raciste.

\section{L'esthétique de la violence}

S'il est vrai que quelques rares penseurs - Hermann Hesse, Romain Rolland, Heinrich Mann, Gustav Landauer, Jean Giono - résistent aux sirènes bellicistes, l'idée d'un fossé séparant les intellectuels et les artistes pacifistes des élites militaires et politiques bellicistes est trompeuse. Les chansons sont le reflet des conjonctures. Elles trahissent bien l'esprit mobilisé, mais reflètent aussi la fatigue et le ras-le-bol de la guerre (Janina Arndts).

\footnotetext{
${ }^{16}$ Annette BeCKer, Les cicatrices rouges. France et Belgique occupées, Paris, Fayard, 2010 ; Philippe NIVET, La France occupée : 1914-1918, Paris, A. Colin, 2011.
} 
Les avant-gardes qui défient les normes bourgeoises et suscitent l'intérêt chez le voisin, jouent souvent avec la violence. La guerre orchestre la transition du " chaud " vers le «froid ", des expressionnistes vers le dadaïsme, de la Kunstgewerbeschule de Van de Velde au Bauhaus. Chez les avant-gardistes qui font beaucoup parler d'eux durant la guerre, comme Dada à Zurich, 1916-17 marque aussi une césure, un tournant. Ce sont souvent d'anciens expressionnistes appelant de leurs vœux la fin du vieux monde. Tout en dénonçant la guerre entre les nations, les révolutionnaires insistent sur la nécessité d'en passer par la violence. Ils ont en commun la conviction qu'il faut se situer sur un terrain tierce dissolvant les antagonismes francoallemands au moyen d'un autre antagonisme. La violence devient ainsi médiatrice, d'une façon qui est toutefois nouvelle.

L'engouement de Guillaume Apollinaire pour la guerre est moins un patriotisme français et une esthétisation de la guerre qu'une tentative désespérée d'humaniser les horreurs au moyen de formes littéraires novatrices (Joseph Jurt). Il est fallacieux d'opposer aux textes de Jünger et Montherlant célébrant la guerre les textes pacifistes d'un Barbusse (Le Feu), d'un Dorgelès (Les Croix de bois), d'un Jean Giono (Le Grand Troupeau), d'un Arnold Zweig (Grischa) ou d'un Remarque (À l'Ouest rien de nouveau). La représentation des horreurs semble créer une symétrie franco-allemande. Cendrars exprime sa haine à l'encontre de la machine de guerre et contre ceux qui ne combattent pas. Seule une écriture obéissant à une nouvelle esthétique agonale, au-delà du beau et du laid, permet de tenir compte des agonalités meurtrières de la guerre. Les textes de Jünger, Cendrars, Genevoix et d'autres traduisent moins les spectacles grandioses des batailles de matériel que les horreurs de la guerre. Les conflits entre soldats de nations différentes font place à ceux entre soldats et civils (Jochen Mecke).

Dans ses écrits de guerre, Robert Musil essaie de bannir l'ébranlement que provoquent les cruautés. Il évite la représentation immédiate du vécu. Par le biais d'un étrange déplacement, la mort des animaux reflète les horreurs de la guerre (Claus Erhart). Les écrits d'Ernst Jünger, de Joseph Roth, de Robert Musil et de Siegfried Kracauer témoignent tous, certes d'une façon très différente, des traumatismes subis. Jünger en rend compte à l'aide d'un art de la prothèse. Roth crée un hiatus entre la photo et la femme réelle. Musil réduit l'écart entre l'homme et l'animal. Ces analyses estompent les différences entre les positions bellicistes et pacifistes (Dorothee Kimmich).

Cependant ces textes soulèvent les critiques de contemporains ou suscitent leurs réserves. La critique de À l'Ouest rien de nouveau se fait d'abord au nom de la vérité historique, l'éditeur ayant imposé des ajouts inventés. Les avant-gardistes, par exemple Mynona/ Friedlaender en Allemagne et Breton en France, reprochent à ces textes de ne pas rompre avec la codification nationale des hostilités et de la violence. L'indifférentiste est le tiers qui refuse le parti pris pour un camp. Il crée un lien franco-allemand transitoire. L'apparition du témoin mêlant attributs français et allemands lors du procès Barrès dément la dualité belligène fatale. L'avant-garde met en scène un 
spectacle qui prend le contrepied de la cérémonie du Soldat inconnu nouvellement instaurée (Thomas Keller).

Si l'esthétique de la violence transfrontalière est provoquée par la guerre franco-allemande, l'exaspération de la violence dissout les deux pôles de l'antagonisme.

\section{La mémoire - témoignage et source de conflits}

La publication de témoignages par Jean Norton Cru (Témoins, essai d'analyse et de critique des souvenirs de combattants édités en français de 1915 à 1928) traduit une crise de la représentation. Pour ce collectionneur acharné de récits véridiques, les romans de Remarque et de Barbusse sont sans valeur historique et même mensongers ${ }^{17}$. La littérature représente les Allemands comme des barbares ou, comme le roman de Remarque, elle se concentre à l'excès sur le macabre. Pour Cru, la qualité d'une écriture de la guerre se mesure à sa volonté de servir la paix et de se mettre au service des soldats. La violence est contradictoire : elle transforme les hommes en ennemis ; la machine de guerre amène les soldats des pays différents à se ressembler; la contrainte exercée par les pouvoirs sur les combattants français et allemands (ou encore britanniques, américains et russes) fait d'eux les victimes de leur propre camp. Les critères de Cru sont sévères : pour avoir droit à la parole, il faut avoir l'expérience du front. Être infirmier ne suffit pas. Le vécu des tranchées, lui, fait nécessairement sortir du cadre officiel. Jusqu'à aujourd'hui, l'éthique du témoignage entre en contradiction avec les historiographies qui s'appuient, comme l'École de Péronne, sur le consentement des soldats au sort qui leur est fait (Jean-Marie Guillon).

Contrairement à un discours très répandu, la mémoire ne désamorce pas le conflit. Elle est toujours du côté des forces coercitives lorsqu'elle sépare deux mémoires, celle des vainqueurs étant forcément différente de celle des vaincus. La " Grande Guerre » n'existe que pour une certaine France. Les lieux de mémoire de la Première Guerre mondiale - le Chemin des Dames, Douaumont, Péronne - se trouvent tous sur le sol français. Si l'instauration de la cérémonie du Soldat inconnu, célébrée sous l'Arc de Triomphe, suscite tout de suite la réprobation des futurs surréalistes Tzara, Max Ernst et André Breton, les anciens combattants semblent pourtant élaborer une culture mémorielle commune. Les rencontres entre anciens combattants français et allemands en témoignent. Le Locarno des poilus est pourtant fallacieux. Leur pacifisme intégral mène à des compromissions, comme leurs contacts avec l'Allemagne nazie ${ }^{18}$.

\footnotetext{
17 Pierre SchoentJes, Fictions de la Grande Guerre. Variations littéraires sur 14-18, Paris, Classiques Garnier, 2009.

${ }_{18}$ Patrick DE VILLEPIN, « Le pacifisme intégral et l'Allemagne nazie (1933-1939) », in Hans Manfred Bock, Reinhart Meyer-KalKus, Michel Trebitsch (dir.), De Locarno à
} 
La culture mémorielle officielle ne réussit pas et n'est pas prête à intégrer les morts comme le montre la pièce de Hans Chlumberg Wunder um Verdun. Le miracle de la résurrection des morts est encombrant pour l'ordre de l'après-guerre. La mémoire collective, les cérémonies de la victoire et le tourisme sur les champs de bataille continuent de pousser à la guerre. Les vivants qui s'identifient aux morts contribuent à renforcer les conflits. Ils obligent les victimes à reproduire des caractéristiques nationales. Les morts se dérobent finalement à jamais à la logique fonctionnelle et nationale (Christa Karpenstein-Essbach).

La conviction que la commémoration interdit le deuil personnel et occulte la mort a conduit les historiens Stéphane Audoin-Rouzeau, Jean-Jacques et Annette Becker, à fonder un " Historial (et non un Mémorial) de la Grande Guerre » à Péronne (Somme, Picardie). Ils expriment ainsi leur scepticisme à l'égard de la commémoration collective. Le deuil doit rester individuel. Bien qu'il s'agisse de la dernière victoire univoque pour les Français, la victoire en elle-même a perdu de son importance. Il faut attendre plus d'un demi-siècle avant que ne soient produits des films, comme La Vie et rien d'autre (1988) de Bertrand Tavernier, ou que ne paraissent des romans, comme Les Champs d'honneur (1990) de Jean Rouaud, qui montrent ce deuil individuel.

Aucun équivalent n'existe dans la littérature ou dans le cinéma d'OutreRhin. La Première Guerre mondiale reste un événement historique plus marquant pour les Français que pour les Allemands. Le film de Christian Caron Joyeux Noël (2005), une co-production française, britannique et allemande sur la trêve de Noël en 1914, n'y change rien. Cette dissymétrie est due surtout à la commémoration de la Shoah qui estompe en Allemagne les deuils antérieurs. Il s'ensuit qu'il est inapproprié, voire impossible, de commémorer en 2014 les années 1914 et 1944 dans un même élan, la Première Guerre Mondiale et la Libération. L'assimilation des deux guerres comporte un risque « de brouillage pour les autres pays déjà impliqués dans la préparation du centenaire, en particulier vis-à-vis de l'Allemagne, qui ne peut se retrouver dans un tel mélange $»^{19}$.

Tandis que la guerre de 14-18 est partiellement effacée de la mémoire collective allemande, elle est devenue l'objet d'une recherche francoallemande intense incarnée par des chercheurs comme Gerd Krumeich et S. Audoin-Rouzeau. Il est toutefois permis de douter que la guerre de 191418, la catastrophe initiale de la guerre civile européenne, représente un événement qui habiterait une mémoire collective européenne.

Deux ouvertures récentes sont à noter: Lionel Jospin, puis Nicolas Sarkozy, ont honoré la mémoire des fusillés pour l'exemple. Les cérémonies sur le sol français connaissent par ailleurs un élargissement dans le cadre du « couple franco-allemand ». Le 22 septembre 1984, François Mitterrand et

Vichy. Les relations culturelles franco-allemandes dans les années 1930, vol. I, Paris, CNRSÉditions, 1993, p. 161-174.

${ }^{19}$ Nicolas OfFENSTADT, Le Monde, 27 octobre 2012, p. 2. 
Helmut Kohl ont uni leurs mains à Verdun. Puis, le 11 novembre 2009, Nicolas Sarkozy et Angela Merkel ont déposé ensemble une gerbe sur la tombe du Soldat inconnu sous l'Arc de Triomphe. Mais ce partage concerne uniquement la fonction matérielle; les gestes symboliques sont impuissants à réunir les deux mémoires. Tout comme Versailles, le 11 novembre 1918 ne peut symboliser la réconciliation franco-allemande (Johannes Grossmann).

Les conflits de la Première Guerre mondiale ne se déroulent pas uniquement dans les tranchées. Les colonies et les territoires occupés dans le Nord de la France deviennent des lieux de combats discursifs. Le déplacement des archives des colonies allemandes pendant et après la guerre reflète la tentative de garder la maîtrise de l'interprétation de la pratique coloniale (Isabell Scheele).

Dans les colonies, les contacts germano-franco-africains créent des configurations triangulaires: la population locale est objet de deux puissances coloniales qui rivalisent entre elles. Elle peut rejeter les deux puissances ou arbitrer. Une femme non-conformiste comme Lucie Couturier peut ainsi se faire l'avocate de la cause africaine et s'opposer au colonialisme. C'est encore un tiers, le britannique Richard $\mathrm{Cobb}^{20}$, qui souligne combien l'interculturalité s'intensifie pendant la guerre (Hans-Jürgen Lüsebrink).

Le poilu déplacé de l'autre côté de la frontière semble déranger également les communautés nationales. Les prisonniers de guerre rentrés en France font l'objet d'un rejet. Siegfried von Kleist alias Jacques Forestier, le poilu devenu allemand dans le roman et la pièce de Giraudoux, retrouve finalement son pays natal. Les transformations et mélanges s'avèrent en fin de compte impossibles. Ernst Erich Noth, écrivain allemand fuyant le nazisme et exilé en France, fait naître un enfant de l'union entre le prisonnier de guerre provençal Paul et la fermière allemande Marie, mais l'enfant se suicide dès qu'il apprend la vérité sur son origine (Thomas Lange). Le contact physique déclenche une crise. L'enfant est encore une fois sacrifié. Il paie de sa vie les conflits entre les parents.

\section{Au-delà de la binarité}

Le chercheur qui établit les liens entre la guerre, la culture et la violence met en question le point de vue binaire. La Première Guerre mondiale, qui semble être la troisième dans la série des quatre guerres franco-allemandes symbolisées par les batailles d'Iéna, Sedan, Verdun et Berlin, marque la dissolution définitive d'une dualité et fond le conflit franco-allemand dans un ensemble plus vaste. Les guerres mondiales brouillent la distinction entre vainqueurs et vaincus. Une certaine écriture de l'histoire donne pourtant

\footnotetext{
${ }^{20}$ Richard COBB, French and Germans, Germans and French. A personal interpretation of France under two occupations, 1914-1918 / 1940-1944, Lebanon, University Press of New England, 1983.
} 
toujours l'impression que Français et Allemands sont, au moins jusqu'en 1945, inexorablement liés par une série de guerres, allant d'Iéna à Berlin, qui départagent chaque fois vainqueur et vaincu. Cette perspective téléologique crée une continuité problématique entre les deux guerres mondiales. L'idée d'un cheminement inéluctable caractérisé par l'antagonisme franco-allemand et culminant dans l'opposition entre la barbarie nazie et la résistance fait de la Première Guerre mondiale un simple maillon dans une chaîne de conflits. Si les deux guerres mondiales sont liées entre 1914 et 1945 par des antagonismes idéologiques qu'on désigne du nom de "guerre civile européenne », le racisme nazi garde tout de même sa spécificité.

L'Europe connaît la paix depuis maintenant plus de soixante ans. Les dynamiques cherchant à régler les conflits de manière rationnelle n'ont pas cessé d'être renforcées (le droit, le traité, le libre commerce, la négociation, la communication...). En témoignent l'ONU, le GATT, puis la WHO, la Communauté Européenne, la législation internationale qui a pour but de définir le génocide et punir les crimes de guerre et les crimes contre l'humanité (procès de Nuremberg, Cour Pénale Internationale de la Haye) et la Cour Européenne des Droits de l'Homme de Strasbourg. Mais il ne peut malgré tout être question d'un monde où les conflits seraient réglés, où toutes les revendications auraient été frappées de nullité. Bien au contraire.

La fin de la nouvelle Guerre de Trente Ans (1914-1945), comme celle de la Guerre froide (1945-1989), n'a pas débouché sur la fin de l'histoire ou sur la paix perpétuelle. Notre époque est une période de dégel des systèmes pacificateurs, de dégel de systèmes politiques et économiques figés, de dégel de l'équilibre de la terreur. Notre présent voit le retour de guerres, mais de guerres nouvelles, de guerres dissymétriques surtout. Les confrontations militaires actuelles ont souvent tendance à vouloir réduire les guerres à des conflits limités, possibles et acceptables, qui évitent l'élargissement à l'échelle mondiale et le risque d'une déflagration nucléaire.

Néanmoins, la mondialisation engendre à son tour des tensions. Il est redevenu habituel de voir les cercueils de jeunes Européens tués dans des combats censés opposer les cultures dites universelles aux cultures dites différentielles. S'il s'avère que les démocraties ne mènent pas de guerre entre elles, l'homogénéisation ne semble pas à même d'apaiser les conflits. La paix que connaît l'Europe depuis 1945 indique apparemment une sortie définitive de la structure infanticide. Toutefois, si une guerre entre Français et Allemands paraît aujourd'hui peu probable, d'autres conflits intra-européens, entre Nord et Sud, entre possédants et dépossédés, se profilent à l'horizon. L'approche choisie ici, qui consiste à retracer le lien entre culture et violence, ne signifie pas que l'on aurait une lecture belliciste de l'histoire. Il ne s'agit pas non plus de renier, de discréditer ou de ridiculiser les mécanismes interétatiques destinés à éviter ou pour le moins à contenir les conflits. Mais les sujets abordés dans ce recueil traduisent un certain scepticisme à l'égard d'une conception trop optimiste et angélique de l'histoire, qui oppose les politiciens et les militaires aux «bons" représentants de la culture. L'Ocell 
THOMAS KELLER

de foc (M. Ernst), l'oiseau de feu, la créature horrible et auto-déstructrice, mihomme, mi-animal, c'est nous.

\section{Remerciements}

Ce recueil réunit les contributions au colloque « La Première Guerre mondiale un siècle plus tard. Culture et violence » qui s'est tenu du 14 au 16 mars 2013 à Aix-en-Provence. Il a bénéficié du soutien de l'Université d'Aix-Marseille (Conseil Scientifique et EA ECHANGES), de l'Université Franco-Allemande, du Conseil départemental des Bouches-du-Rhône et du Centre Franco-Allemand de Provence. Que toutes ces institutions soient chaleureusement remerciées.

\section{Bibliographie}

\section{Textes de guerre}

Bergson, Henri, La signification de la guerre, Paris, Bloud et Gay, 1919.

BERR, Henri, Le germanisme contre l'esprit français, Paris, La Renaissance du livre, 1919.

CRU, Jean-Norton, Témoins, essai d'analyse et de critique des souvenirs de combattants édités en français de 1915 à 1928, Paris, Les Étincelles, 1929 [rééd. Presses Universitaires de Nancy, 1993].

DURKheIM, Emile, L'Allemagne au-dessus de tout, Paris, A. Colin, 1915 [repr. La mentalité allemande et la guerre, Paris, A. Colin (coll. " L'Ancien et le Nouveau »), 1991.

Plenge, Johann, Der Krieg und die Volkswirtschaft, Münster, Borgmeyer, 1915 ; id., Eine Kriegsvorlesung über die Volkswirtschaft. Das Zeitalter der Volksgenossenschaft, Berlin, Springer, 1915 ; id., 1789 und 1914. Die symbolischen Jahre in der Geschichte des politischen Geistes, Berlin, Springer, 1916.

RIVIÈRE, Jacques, L'Allemand, souvenirs et réflexions d'un prisonnier de guerre, Paris, NRF, 1918 [rééd. 1924].

SCheler, Max, Der Genius des Krieges und der Deutsche Krieg, Leipzig, Die Weissen Bücher, 1915.

« Aufruf an die Kulturwelt » (1914), in VOM BRUCH, Rüdiger, HOFMEISTER, Björn (éds.), Deutsche Geschichte in Quellen und Darstellung, vol. VIII, Stuttgart, Reclam, 2000, p. $366-369$.

\section{Littérature critique}

Audoin-RouzeAu, Stéphane, BECKER, Annette, Ingrao, Christian, Rousso, Henry (dir.), La violence de la guerre 1914-1945, Paris, Ed. Complexe, 2002.

Audoin-Rouzeau, Stéphane, KrumeICH, Gerd, Cicatrices : paysages de guerre, 1914-1918, photographies Jean Richardot, Paris, Tallandier, 2008.

BEAUPRÉ, Nicolas, Écrire en guerre, écrire la guerre (France, Allemagne 1914-1920), Paris, CNRS Éditions, 2006 ; id., Deutsch-französische Geschichte (Histoire franco- 
allemande), vol. VIII, Das Trauma des großen Krieges (1918-1932/33), Darmstadt, Wissenschaftliche Buchgesellschaft, 2009 ; id., Les Grandes Guerres 1914-1945, Paris, Belin, 2012.

BECKER, Annette, Oubliés de la Grande Guerre. Humanitaire et culture de guerre, Paris, Noésis, 1998 [rééd. 2003] ; id., Apollinaire et la guerre. Une biographie de la guerre 1914-1918, Paris, Talandier, 2009 ; id., Les cicatrices rouges. France et Belgique occupées, Paris, Fayard, 2010.

BECKER, Jean-Jacques, KRUMEICH, Gerd, La Grande guerre. Une histoire franco-allemande, Paris, Tallandier, 2008 (Der Große Krieg. Deutschland und Frankreich 1914-1918, Essen, Klartext Verlag, 2010).

BESSLICH, Barbara, Wege in den Kulturkrieg. Zivilisationskritik in Deutschland 1890-1914, Darmstadt, Wissenschaftliche Buchgesellschaft, 2000.

Borso, Vittória, Krumeich, Gerd, Witte, Bernd (dir.), Medialität und Gedächtnis. Interdisziplinäre Beiträge zur kulturellen Verarbeitung europäischer Krisen, Stuttgart/ Weimar, Metzler, 2001.

BRÖCKLING, Ulrich, SIKORA, Michael (dir.), Armeen und Deserteure. Vernachlässigte Kapitel einer Militärgeschichte der Neuzeit, Göttingen, Vandenhoeck \& Ruprecht, 1998.

CAHN, Jean-Paul, KNOPPER, Françoise, SAINT-GILlE, Anne-Marie (dir.), De la guerre juste à la paix juste. Aspects confessionnels de la construction de la paix dans l'espace francoallemand (XVI'-XX siècle), Villeneuve d'Ascq, Presses Universitaires du Septentrion, 2008.

CePl-Kaufmann, Gertrude, Krumeich, Gerd, Sommers, Ulla (dir.), Krieg und Utopie. Kunst, Literatur und Politik im Rheinland nach dem Ersten Weltkrieg, Essen, Klartext Verlag, 2006.

ClARK, Christopher, The Sleepwalkers. How Europe went to war in 1914, Londres, Penguin, 2012.

COBB, Richard, French and Germans, Germans and French. A personal Interpretation of France under two occupations, 1914-1918/ 1940-1944, Lebanon, University Press of New England, 1983.

Curtius, Ernst Robert, Die literarischen Wegbereiter des neuen Frankreich, Potsdam, G. Kiepenheuer, 1923.

DAGAN, Yaël, La NRF entre guerre et paix 1914-1925, Paris, Tallandier, 2008.

DetUE, Frédéric, « Le schisme littéraire des témoignages de la Grande Guerre », Vox Poetica. Lettres et sciences humaines, consultable sur : http://www.vox-poetica.org/t/articles/ detue2013.html.

Dülffer, Jost, KrumeICH, Gerd, (dir.), Der verlorene Frieden. Politik und Kriegskultur nach 1918, Essen, Klartext Verlag, 2002.

FLASCH, Kurt, Die geistige Mobilmachung. Die deutschen Intellektuellen und der Erste Weltkrieg, Ein Versuch, Berlin, Alexander Fest, 2000.

FrANÇOIS, Étienne, «Die Gegenwart des Vergangenen. Der Erste Weltkrieg in der zeitgenössischen französischen Literatur », in Roswitha BÖHM, Stephanie BUNG, Andrea GREWE (dir.), Observatoire de l'extrême contemporain, Studien zur französischsprachigen Gegenwartsliteratur, Tübingen, Narr, 2009, p. 115-130.

FRIES, Helmut, Die große Katharsis. Der Erste Weltkrieg in der Sicht deutscher Dichter und Gelehrter, 2 vol., Constance, Verlag am Hockgraben, 1994-95.

HoEREs, Peter, Krieg der Philosophen. Die deutsche und die britische Philosophie im Ersten Weltkrieg, Paderborn, Schöningh, 2004.

HORNE, John (dir.), Vers la guerre totale. Le tournant de 1914-15, Paris, Tallandier, 2000.

Hull, Isabell V., Absolute destruction : Military Culture and the Practices of War in Imperial Germany, Ithaka, Cornell University Press, 2004. 
JAHR, Christoph, Gewöhnliche Soldaten. Desertion und Deserteure im deutschen und britischen Heer 1914-1918, Göttingen, Vandenhoeck \& Ruprecht, 1998.

JeIsmann, Michael, Das Vaterland der Feinde, Stuttgart, Klett-Cotta, 1992 (La patrie de l'ennemi. La notion d'ennemi national et la représentation de la nation en Allemagne et en France de 1792 à 1918, Paris, CNRS Éditions, 1997).

JONES, Heather, Violence against prisoners of war in the First World War. Britain, France, Germany, 1914-1920, Cambridge, Cambridge University Press, 2011.

KARPENSTEIN-ESSBACH, Christa, Orte der Grausamkeit. Die neuen Kriege in der Literatur, München, Fink, 2011 ; id., « Krieg und Geschichte. Zur literarischen Repräsentation des Ersten Weltkrieges im Ausgang der Weimarer Republik », in Elisabeth GuILHAMON, Daniel MeYer (dir.), Die streitbare Klio. Zur Repräsentation von Macht und Geschichte in der Literatur, Frankfurt am Main/ Berlin, Lang, 2010, p. 109-126.

Keller, Thomas, Deutsch-Französische Dritte-Weg-Diskurse. Personalistische Debatten der Zwischenkriegszeit, München, Fink, 2001.

KNOPPER, Françoise, «Les traités de paix, des tournants? », Revue d'Allemagne et des pays de langue allemande, 2011, p. 163-318.

KRUMEICH, Gerd (dir.), Versailles 1919, Essen, Klartext Verlag, 2001.

Krumeich, Gerd, BRANDT, Susanne, Schlachtenmythen. Ereignis - Erzählung - Erinnerung, Bonn, Bouvier, 2003 (Europäische Geschichtsdarstellungen, 2).

LE NAOUR, Jean-Yves, Fusillés. Enquête sur les crimes de la justice militaire, Paris, Larousse, 2010.

LEONHARD, Jörn, Bellizismus und Nation, Kriegsdeutung und Nationsbestimmung, Europa und die Vereinigten Staaten 1750-1914, München, Oldenbourg, 2008.

Majerus, Benoît, Kmac, Sonja, Largue, Michel, PÉPorté, Pit (dir.), Dépasser le cadre national des lieux de mémoire/ Nationale Erinnerungsorte hinterfragt, Bruxelles, Lang, 2009.

MARIOT, Nicolas, Tous unis dans la tranchée? 1914-1918. Les intellectuels rencontrent le peuple, Paris, Seuil, 2013.

NiVET, Philippe, La France occupée : 1914-1918, Paris, A. Colin, 2011.

OFFENSTADT, Nicolas, 14-18 aujourd'hui. La Grande Guerre dans la France contemporaine, Paris, Odile Jacob, 2010 ; id., Les fusillés de la grande guerre et la mémoire collective (1914-1999), Paris, Odile Jacob, 1999.

PFETSCH, Frank R. (dir.), Konflikt, Berlin, Springer, 2004.

PINKER, Steven, Gewalt. Eine neue Geschichte der Menschheit, Frankfurt, Fischer, 2011.

POLlet, Jean-Jacques, SAINT-Gille, Anne-Marie (dir.), Écritures franco-allemandes de la Grande Guerre, Arras, Artois Presses Université, 1996.

Prochasson, Christophe, Rasmussen, Anne, Au nom de la patrie, les intellectuels et la première guerre mondiale 1910-1919, Paris, La Découverte, 1996.

Prost, Antoine, WINTER, Jay, Penser la Grande Guerre : un essai d'historiographie, Paris, Seuil, 2004.

PRÜMM, Karl, Die Literatur des soldatischen Nationalismus der 20er Jahre. Gruppenideologie und Epochenproblematik, 2 vol., Kronberg/ Taunus, Scriptor Verlag, 1974.

Pyta, Wolfram, KRETSChMANN, Carsten (dir.), Burgfrieden und union sacrée. Literarische Deutungen und politische Ordnungsvorstellungen in Deutschland und Frankreich 19141933, München, Oldenbourg, 2011.

RiEUNEAU, Maurice, Guerre et révolution dans le roman français de 1919 à 1939, Paris, Klincksieck, 1974 [rééd. 2000].

SCHILDT, Axel, « Ein konservativer Prophet moderner nationaler Integration. Biographische Skizze des streitbaren Soziologen Johann Plenge », Vierteljahrshefte für Zeitgeschichte, $\mathrm{n}^{\circ} 35,1987$, p. $523-570$. 
SCHIVELBUSCH, Wolfgang, Die Kultur der Niederlage. Der amerikanische Süden 1865, Frankreich 1871, Deutschland 1918, Berlin, Alexander Fest Verlag, 2001.

SCHLOTTER, Sven, Die Totalität der Kultur. Philosophisches Denken und politisches Handeln bei Bruno Bauch, Würzburg, Königshausen \& Neumann, 2004.

SCHÖNING, Matthias, Versprengte Gemeinschaft. Kriegsroman und intellektuelle Mobilmachung in Deutschland 1914-1933, Göttingen, Vandenhoeck \& Ruprecht, 2009.

SCHNEIDER, Sebastian, « Der Krieg als eine besondere Form des Konflikts », in Trajectoire, $\mathrm{n}^{\circ}$ 5, 2011, Repenser le conflit, consultable en ligne http://trajectoires.revues.org/770.

SCHNEIDER, Uwe, SCHUMANN, Andreas (dir.), "Krieg der Geister». Erster Weltkrieg und literarische Moderne, Würzburg, Könighausen \&Neumann, 2000.

SERres, Michel, Petites chroniques du dimanche soir 2, février 2006 - mars 2007, Paris, Le Pommier, 2007, p.30-31.

SNYDER, Timothy, The better angels of our nature. Why violence declined, New York, Barnes and nobles, 2011.

StANZEL, Franz K., LÖSCHNIGG, Martin (dir.), Intimate Enemies. English and German Literary Reactions to the Great War 1914-1918, Heidelberg, Winter, 1993.

VERHEY, Jeffrey, Der « Geist von 1914 » und die Erfindung der Volksgemeinschaft, Hamburg, Hamburger Edition, 2000.

Vondung, Klaus (dir.), Kriegserlebnis. Der Erste Weltkrieg in der literarischen Gestaltung und symbolischen Deutung der Nationen, Göttingen, Vandenhoeck \& Ruprecht, 1980.

VON UNGERN-STERNBERG, Jürgen, « Wie gibt man dem Sinnlosen einen Sinn ? Zum Gebrauch der Begriffe "deutsche Kultur" und "Militarismus" im Herbst 1914 », in Wolfgang J. MommSEn (dir.), Kultur und Krieg. Die Rolle der Intellektuellen, Künstler und Schriftsteller im Ersten Weltkrieg, München, Oldenbourg, 1996, p. 76-96.

VON Ungern-STERnBERG, Jürgen, Von UngERn-STERnBERG, Wolfgang, Der Aufruf « An die Kulturwelt! ». Das Manifest der 93 und die Anfänge der Kriegspropaganda im Ersten Weltkrieg. Mit einer Dokumentation, Stuttgart, Reclam, 1996.

WERBER, Niels, «Erzählen im Krieg und Nicht-Krieg », LiLi, Zeitschrift für Literaturwissenschaft und Linguistik, $42^{\mathrm{e}}$ année, $\mathrm{n}^{\circ}$ 165, Postsouveränes Erzählen, mars 2012, p. 87-105. 